\title{
Some remarks concerning rank 2 bundles and Chow groups
}

\author{
By $K$. Hulek at Hannover and $A$. Van de Ven at Leiden
}

\section{§0. Introduction}

To every algebraic vector bundle $F$ on a (smooth, projective) variety $X$ one can associate Chern classes $c_{i}=c_{i}(F) \in A^{i}(X)$ where $A^{i}(X)$ denotes the Chow group in codimension $i$. The case $i=1$ shows that these are much finer invariants than the topological Chern classes $c_{i}^{\text {top }}(F) \in H^{2 i}(X, \mathbb{Z})$. Now if $S$ is a surface, then given two classes $c_{i} \in A^{i}(S)$; $i=1,2$ there always exists a rank 2 vector bundle $F$ on $S$ with $c_{i}(F)=c_{i}$. This is easy to see, but there seems to be no reference in the literature. (The corresponding topological result was proved by Schwarzenberger in [Sch].) In general it is of course not possible to give the classes $c_{i}$ arbitrarily since the topological classes determined by the $c_{i}$ have to be the Chern classes of an algebraic bundle. Even if this is the case it seems unlikely that there always exists a bundle with given classes $c_{i}$. But it does not seem easy to construct counterexamples. In this paper we consider the question from the point of view of deformations. More precisely, we present two examples showing that the answer to the following question of $\mathrm{Ph}$. Griffiths is negative.

Question. Let $X$ be a variety and let $F$ be a vector bundle on $X$ with Chern classes $c_{i}=c_{i}(F) \in A^{i}(X)$. Moreover let $\mathscr{X}=\left(X_{t}\right)_{t \in T} \rightarrow T$ be a deformation of $X$ such that the Chern classes of $F$ can be extended to $\mathscr{X}$ (i.e. there are varieties $Z_{i j} \subset \mathscr{X}$ and integers $n_{i j}$, such that $Z_{i j} \cap X_{t}=Z_{i j, t}$ is defined for all $t$, whereas $\sum_{j} n_{j} Z_{i j, 0}$ represents $c_{i}$ ). Can one then extend $F$ to a vector bundle $\mathscr{F}$ on $\mathscr{X}$ ?

Here we present two counterexamples, one in the case where $X$ is a surface $(\S 2)$, the other where $X$ is a threefold ( $(3)$. In both cases $F$ has rank 2. These two examples have very different flavours. In the surface case it is trivial that $c_{2}$ can be extended to the deformation $\mathscr{X}$. In the case of threefolds this is a highly non-trivial condition. The second example (we use a sextic threefold in $\mathbb{P}_{4}$ ) is much more subtle and we believe it to have some intrinsic geometric interest. 


\section{§ 1. Rank 2 bundles on surfaces}

In this section we prove

Proposition 1. Let $S$ be a smooth projective surface. For given Chern classes $c_{i} \in A^{i}(S)$; $i=1,2$ there exists a rank 2 vector bundle $F$ on $S$ with $c_{i}(F)=c_{i} ; i=1,2$.

Proof. Let $H$ be a very ample divisor on $S$. For $n \in \mathbb{N}$ we consider the Chern classes

$$
\begin{aligned}
& \tilde{c}_{1}:=c_{1}+2 n H, \\
& \tilde{c}_{2}:=c_{2}+n H . c_{1}+n^{2} H^{2} .
\end{aligned}
$$

By Kodaira vanishing we have

$$
h^{2}\left(\tilde{c}_{1}\right)=h^{2}\left(c_{1}+2 n H\right)=0 \text { for } n \gg 0 .
$$

By $[\mathrm{Kl}]$, theorem 5.8 , we can write

$$
c_{1}=D-E
$$

where $D, E$ are smooth curves. Hence in $A^{2}(S)$ :

i.e.

$$
\tilde{c}_{2}=c_{2}+n H(D-E)+n^{2} H^{2}
$$

$$
\tilde{c}_{2}=c_{2}+n H^{2}+n H[(n-1) H+D-E] .
$$

Using Kleiman-Hironaka's result on smoothing cycles [K1], theorem 5.8, we can write for $n$ sufficiently big

$$
c_{2}+n H^{2}=Z_{1}
$$

where $Z_{1}$ is a smooth cycle. Again for $n$ big enough we have

$$
n H[(n-1) H+D-E]=Z_{2}
$$

where $Z_{2}$ is smooth and disjoint from $Z_{1}$. Hence $\tilde{c}_{2}$ can be represented by the smooth cycle $Z=Z_{1}+Z_{2}$. Because of (1) we can apply Serre's construction [OSS], §5, and get an extension

$$
0 \rightarrow \mathcal{O}_{S} \rightarrow E \rightarrow \mathscr{I}_{Z}\left(-c_{1}-2 n H\right) \rightarrow 0
$$

where $E$ is a vector bundle with Chern classes

$$
\begin{aligned}
& c_{1}(E)=-c_{1}-2 n H=-\tilde{c}_{1}, \\
& c_{2}(E)=Z=\tilde{c}_{2} .
\end{aligned}
$$

It follows that the vector bundle 


$$
F:=E^{*} \otimes \mathcal{O}_{S}(-n H)
$$

has the desired Chern classes, i.e. $c_{i}(F)=c_{i}$ for $i=1,2$.

\section{§2. Surface case}

Here we give an example which provides a negative answer to Griffiths' question in the case where $S$ is a projective surface and $F$ is a rank 2 bundle. We start with a Comessatti surface $S \subset \mathbb{P}_{4}$, i.e. an abelian surface whose hyperplane section is of the form

$$
H=C+D
$$

where $C, D$ are smooth genus 2 curves and

$$
\operatorname{deg} C^{2}=\operatorname{deg} D^{2}=2, \quad \operatorname{deg}(C . D)=3 .
$$

For more details about these surfaces see [La]. We now choose a family $\mathscr{X}=\left(X_{t}\right)_{t \in T}$ of abelian surfaces in $\mathbb{P}_{4}$ such that

$$
\operatorname{Num}\left(X_{t}\right)=\mathbb{Z} H
$$

for generic $t \in T$ (i.e. there is a dense subset of $t$ with this property). We set

Then

$$
F=\mathcal{O}_{x}(-C) \oplus \mathcal{O}_{x}(C)
$$

$$
c_{1}(F)=0, \quad c_{2}(F)=-C^{2} .
$$

These Chern classes can be extended to $\mathscr{X}$. This is trivial for $c_{1}$. For $c_{2}$ this follows since $c_{2}(F)$ can be represented as the difference of two smooth cycles (cf. the proof of proposition 1).

Proposition 2. The vector bundle $F$ cannot be extended to $\mathscr{X}$.

Proof. Assume there is an extension $\mathscr{F}$ of $F$ to $\mathscr{X}$ and let

$$
F_{t}:=\mathscr{F} \mid X_{t} \quad(t \in T)
$$

By construction, Riemann-Roch and the ampleness of $C$

$$
\begin{aligned}
& h^{0}(F)=h^{2}(F)=\frac{1}{2} \operatorname{deg} C^{2}=1, \\
& h^{1}(F)=0 .
\end{aligned}
$$

Hence by semi-continuity

$$
h^{0}\left(F_{t}\right)=h^{2}\left(F_{t}\right)=1, \quad h^{1}\left(F_{t}\right)=0
$$

Let $t \in T$ be such that (2) holds. By (3) there exists a section $0 \neq s \in H^{0}\left(F_{t}\right)$. First assume that its zero-set $(s)_{0}$ contains a curve, say $E$. Then 


$$
h^{0}\left(F_{t} \otimes \mathcal{O}(-E)\right)=h^{0}\left(F_{t} \otimes \mathcal{O}\left(-n H^{\prime}\right)\right) \neq 0
$$

where $n \in \mathbb{N}$ and $H^{\prime}$ is numerically equivalent to $H$. But this contradicts semi-continuity since

$$
h^{0}\left(F \otimes \mathcal{O}\left(-n H^{\prime}\right)\right)=0 .
$$

The latter follows e.g. from

$$
\left(-C-n H^{\prime}\right) . H<0, \quad\left(C-n H^{\prime}\right) . H<0 .
$$

Hence $(s)_{0}$ has the expected codimension and we get a contradiction since

$$
\operatorname{deg}(s)_{0}=\operatorname{deg} c_{2}=-\operatorname{deg} C^{2}=-2<0 .
$$

Remarks. (i) Using the above ideas one can, of course, easily construct other examples, similar to the one given here.

(ii) Using the proof of proposition 1 one can construct a vector bundle $F^{\prime}$ with $c_{i}\left(F^{\prime}\right)=c_{i}(F), i=1,2$ which extends to $\mathscr{X}$.

\section{§3. The threefold case}

Before we can give our example we need some preparations. Let $X \subset \mathbb{P}_{4}=\mathbb{P}$ be a smooth sextic hypersurface containing a line $L$ (there exist many examples as we shall see shortly).

From the normal bundle sequence

$$
\left.0 \rightarrow N_{L / X} \rightarrow N_{L / P} \rightarrow N_{X / P}\right|_{L} \rightarrow 0
$$

one finds that $c_{1}\left(N_{L / X}\right)=\mathcal{O}_{L}(-3)$. Since moreover

$$
h^{1}\left(\mathcal{O}_{X}(k)\right)=h^{2}\left(\mathcal{O}_{X}(k)\right)=0 \quad(k \in \mathbb{Z})
$$

Serre's construction gives a (unique) extension

$$
0 \rightarrow \mathcal{O}_{X} \rightarrow F \rightarrow \mathscr{I}_{L / X}(-3) \rightarrow 0
$$

where $F$ is a rank 2 bundle on $X$ with

$$
c_{1}(F)=\mathcal{O}_{X}(-3), \quad c_{2}(F)=L .
$$

Let us now consider a family $\mathscr{X}=\left(X_{t}\right)_{t \in T}$ of sextics in $\mathbb{P}_{4}$ with $X_{0}=X$. We assume $T$ to be Stein.

Proposition 3. $F$ can be extended to $\mathscr{X}$ if and only if $L$ deforms in $\mathscr{X}$, i.e. there is a surface $S \subset \mathscr{X}$ s.th. $L_{t}=S \cap X_{t}$ is a line in $X_{t}$ for all $t \in T$.

Before proving this we first show 
Lemma 1. (i) $h^{0}(F(1))=5$.

(ii) $h^{1}(F(1))=h^{2}(F(1))=0$.

(iii) $h^{3}(F(1))=h^{0}\left(\mathcal{O}_{X}(3)\right)=\left(\begin{array}{l}7 \\ 4\end{array}\right)$.

Proof. (i) follows immediately from (4). We consider the exact sequence

$$
0 \rightarrow \mathscr{I}_{L / X}(k) \rightarrow \mathcal{O}_{X}(k) \rightarrow \mathcal{O}_{L}(k) \rightarrow 0 .
$$

This gives $h^{1}\left(\mathscr{I}_{L / X}(-2)\right)=0$ and from (4) we get $h^{1}(F(1))=0$. By Serre duality

$$
h^{2}(F(1))=h^{1}\left(F^{*}(-1) \otimes K_{X}\right)=h^{1}\left(F^{*}\right)=h^{1}(F(3)) .
$$

Again using (5) we find $h^{1}\left(\mathscr{I}_{L / X}\right)=0$ and (4) then implies $h^{1}(F(3))=0$. Using duality once more we find

$$
h^{3}(F(1))=h^{0}(F(3))
$$

and from (4) we get

$$
h^{0}(F(3))=h^{0}\left(\mathcal{O}_{X}(3)\right)=h^{0}\left(\mathcal{O}_{P}(3)\right)=\left(\begin{array}{l}
7 \\
4
\end{array}\right)
$$

Proof of proposition 3. First assume that $L$ deforms in $\mathscr{X}$. Since $h^{i}\left(\mathcal{O}_{X}(k)\right)=0$ for $i=1,2$ it follows from semi-continuity and the assumption that $T$ is Stein that $h^{i}\left(\mathcal{O}_{x}(k)\right)=0$. Hence we can apply Serre's construction to $S \subset \mathscr{X}$ thus getting a vector bundle $\mathscr{F}$ which extends $F$.

Now assume that $F$ extends to $\mathscr{F}$ and let $F_{t}:=\left.\mathscr{F}\right|_{X_{t}}$. Since $h^{0}(F)=1$ we have two cases, namely (1) $h^{0}\left(F_{t}\right)=0$ for $t \neq 0$ and (2) $h^{0}\left(F_{t}\right)=1$ for all $t \in T$. We claim that (1) cannot occur. It follows from $c_{2}(F)=L$ that $c_{2}\left(F_{t}\right) . H=1$ for all $t \in T$ (here $H$ denotes the hyperplane in $\mathbb{P})$. Hence

$$
c_{2}\left(F_{t}\right)=c_{2}\left(F_{t}^{*}\right)=c_{2}\left(F_{t}(3)\right)=L_{t}
$$

where $L_{t} . H=1$ for all $t$, i.e. $L_{t}$ is the class of a line in projective space. Moreover

$$
c_{2}\left(F_{t}(1)\right)=c_{2}\left(F_{t}(2)\right)=L_{t}-2 H_{X_{t}}^{2}
$$

where $H_{X_{t}}=H \cap X_{t}$. Hence $h^{0}\left(F_{t}\right)=0$ for $t \neq 0$ implies $h^{0}\left(F_{t}(l)\right)=0$ for $l \leqq 2$. In particular $h^{0}\left(F_{t}(1)\right)=0$. By semi-continuity it follows from lemma 1 that $h^{1}\left(F_{t}(1)\right)=h^{2}\left(F_{t}(1)\right)=0$. Again by lemma 1 this shows

$$
h^{0}\left(F_{t}(3)\right)=h^{3}\left(F_{t}(1)\right)=\left(\begin{array}{l}
7 \\
4
\end{array}\right)-5>0 \quad(t \neq 0) .
$$

Since $h^{0}\left(F_{t}(l)\right)=0$ for $l \leqq 2$ every section $0 \neq s \in \Gamma\left(F_{t}(3)\right)$ vanishes in codimension 2 . Since $c_{2}\left(F_{t}(3)\right)=L_{t}$ and since $(s)_{0}$ has no embedded components it follows that $(s)_{0}=L_{t}$ is a line as a scheme. But then we get an extension 


$$
0 \rightarrow \mathcal{O}_{X_{t}} \rightarrow F_{t}(3) \rightarrow \mathscr{I}_{L_{t} / X_{L}}(3) \rightarrow 0
$$

from which one gets that $c_{1}\left(N_{L_{t} / X_{t}}\right)=\mathcal{O}_{X_{t}}(3)$, a contradiction.

It remains to treat case (2). In this case $\pi_{*} \mathscr{F} \cong \mathcal{O}_{T}$ where $\pi: X \rightarrow T$ is the projection map. Consider the section $1 \in \Gamma\left(\mathcal{O}_{T}\right) \cong \Gamma(\mathscr{F})$. It follows from (4) and semicontinuity that $h^{0}\left(F_{t}(-1)\right)=0$ for all $t \in T$. Hence the section constructed above vanishes in codimension 2 for all $t \in T$ and it's zero-set gives the desired surface $S$.

We now choose a smooth quadric surface $Q \subset \mathbb{P}_{3} \subset \mathbb{P}$. On $Q$ we consider lines $L, K$ which belong to different rulings, i.e. $L \sim(1,0), K \sim(0,1)$.

Proposition 4. There exists a sextic $X \subset \mathbb{P}_{4}$ s.th.

(S1) $X$ is smooth.

(S2) $X \cap Q=L \cup 2 K \cup R$ where $R$ is a smooth curve of bidegree $(5,4)$ and $(K \cup R) \cap L=\left\{P_{1}, \ldots, P_{5}\right\}$ consists of mutually distinct points.

Proof. Let $H_{Q}$ be the hyperplane section on $Q$. The restriction map

$$
\text { rest: }|6 H| \rightarrow\left|6 H_{Q}\right|
$$

is surjective. We set

$$
|6 H-2 K-L|:=\text { rest }^{-1}\left|6 H_{Q}-2 K-L\right| .
$$

Our first claim is that $|6 H-2 K-L|$ contains a smooth member. Clearly the base locus of $|6 H-2 K-L|$ is $K \cup L$. By Bertini's theorem the general member of $|6 H-2 K-L|$ is therefore smooth outside $K \cup L$. Hence it is enough to show that for each $P \in K \cup L$ the set of all sextics in $|6 H-2 K-L|$ which are singular at $P$ has codimension $\geqq 2$. For this it suffices to construct a pencil in $|6 H-2 K-L|$ s. th. every member of this pencil is smooth at $P$. This is easy: Let $q \in \Gamma\left(\mathcal{O}_{\mathbb{P}_{3}}(2)\right)$ be an equation for $Q$ and $z_{4} \in \Gamma\left(\mathcal{O}_{P}(1)\right)$ an equation for $\mathbb{P}_{3}$. Moreover let $f_{i} \in \Gamma\left(\mathcal{O}_{P_{3}}(i)\right)$ for $i=4,5$ s.th. $f_{i}(P) \neq 0$. Then the pencil spanned by $q f_{4}$ and $z_{4} f_{5}$ will do.

Now let $R \subset Q$ be any smooth curve of bidgeree $(5,4)$ s.th. $(K \cup R) \cap L=\left\{P_{1}, \ldots, P_{5}\right\}$ with the points $P_{i}$ mutually different. Let $X^{\prime}$ be a sextic with $X^{\prime} \cap Q=L \cup 2 K \cup R$. Then (S2) is fulfilled. Since the general member of the linear system $|6 H-2 K-L|$ is smooth we can deform $X^{\prime}$ slightly to a smooth sextic $X$. Since (S2) is an open condition in $|6 H-2 K-L|$ we are done.

Let $X$ be as above. From the normal bundle sequence

$$
\left.0 \rightarrow N_{L / X} \rightarrow N_{L / \boldsymbol{P}} \rightarrow N_{X / \boldsymbol{p}}\right|_{L} \rightarrow 0
$$

we get a map

$$
\alpha_{X}: H^{0}\left(N_{L / P}\right) \rightarrow H^{0}\left(\left.N_{X / P}\right|_{L}\right)=H^{0}\left(\mathcal{O}_{L}(6)\right)
$$

Let

$$
(K \cup R) \cap L=\left\{P_{1}, \ldots, P_{5}\right\}
$$


Then the vector space

$$
W:=\left\{u \in H^{0}\left(\mathcal{O}_{\mathrm{L}}(6)\right) ; u\left(P_{i}\right)=0, i=1, \ldots, 5\right\}
$$

has dimension 2.

Proposition 5. There exists a sextic $X \subset \mathbb{P}_{4}$ s.th. (S1) and (S2) hold and additionally (S3) $W \nsubseteq \operatorname{Im} \alpha$.

Proof. Note that (S3) is a consequence of

(S3)' The following equations are linearly independent in $H^{0}\left(N_{L / P}\right)$ :

$$
\alpha_{X}(s)\left(P_{i}\right)=0 \quad\left(s \in H^{0}\left(N_{L / P}\right) ; i, \ldots, 5\right) .
$$

Assume we are given $L, K, R$ as in proposition 4. Since $Q$ is smooth and $L \subset Q$ we can introduce homogeneous coordinates $z_{0}, \ldots, z_{4}$ in $\mathbb{P}=\mathbb{P}_{4}$, s.th.

$$
\begin{aligned}
& \mathbb{P}_{3}=\left\{z_{4}=0\right\}, \\
& L=\left\{z_{2}=z_{3}=z_{4}=0\right\}
\end{aligned}
$$

and such that

$$
\begin{cases}\frac{\partial q}{\partial z_{2}}\left(P_{1}\right)=0, & \frac{\partial q}{\partial z_{3}}\left(P_{1}\right) \neq 0 \\ \frac{\partial q}{\partial z_{2}}\left(P_{2}\right) \neq 0, & \frac{\partial q}{\partial z_{3}}\left(P_{2}\right)=0 \\ \frac{\partial q}{\partial z_{2}}\left(P_{3}\right) \neq 0, & \frac{\partial q}{\partial z_{3}}\left(P_{3}\right) \neq 0\end{cases}
$$

Using the coordinates $z_{2}, z_{3}, z_{4}$ we get an identification

$$
H^{0}\left(N_{L / P}\right) \cong 3 H^{0}\left(\mathcal{O}_{L}(1)\right)
$$

Let $X^{\prime}=\left\{f_{6}^{\prime}=0\right\}$ be any sextic in $\mathbb{P}$ through $L$. Then the map

becomes

$$
\alpha_{\mathbf{X}^{\prime}}: H^{0}\left(N_{L / p}\right) \rightarrow H^{0}\left(\left.N_{X^{\prime} / p}\right|_{L}\right)
$$

$$
\begin{aligned}
\alpha_{X^{\prime}}: 3 H^{0}\left(\mathcal{O}_{L}(1)\right) & \rightarrow H^{0}\left(\mathcal{O}_{L}(6)\right), \\
s=\left(s_{2}, s_{3}, s_{4}\right) & \left.\mapsto \sum_{i=2}^{4} \frac{\partial f_{6}^{\prime}}{\partial z_{i}}\right|_{L} \cdot s_{i} .
\end{aligned}
$$

Our first aim is to construct a sextic $X^{\prime}$ through $L$, s.th. the equations

$$
\alpha_{X^{\prime}}(s)\left(P_{i}\right)=0 \quad(i=1, \ldots, 5)
$$


are linearly independent in $3 H^{0}\left(\mathcal{O}_{L}(1)\right)$. To do this we choose polynomials $f_{j} \in \Gamma\left(\mathcal{O}_{P_{3}}(j)\right)$; $j=4,5$ s.th.

$$
\begin{cases}f_{4}\left(P_{i}\right) \neq 0 ; i=1,2,3 ; & f_{4}\left(P_{i}\right)=0 ; i=4,5 \\ f_{5}\left(P_{i}\right)=0 ; i=1,2,3 ; & f_{5}\left(P_{i}\right) \neq 0 ; i=4,5\end{cases}
$$

We consider the sextic $X^{\prime}=\left\{f_{6}^{\prime}=0\right\}$ where

$$
f_{6}^{\prime}=q \cdot f_{4}+z_{4} \cdot f_{5} .
$$

Straightforward calculation shows that the equations (7) then become

$$
\left(\frac{\partial q}{\partial z_{2}} f_{4} \cdot s_{2}+\frac{\partial q}{\partial z_{3}} f_{4} \cdot s_{3}+f_{5} \cdot s_{4}\right)\left(P_{i}\right)=0 \quad(i=1, \ldots, 5) .
$$

Using equations (9) for $i=4,5$ and (8) this implies immediately $s_{4}=0$. The remaining equations become

$$
\left\{\begin{array}{l}
s_{3}\left(P_{1}\right)=s_{2}\left(P_{2}\right)=0 \\
\left(\frac{\partial q}{\partial z_{2}} f_{4} \cdot s_{2}+\frac{\partial q}{\partial z_{3}} f_{4} \cdot s_{3}\right)\left(P_{3}\right)=0
\end{array}\right.
$$

Because of (6) these equations are clearly independent and we have found the desired $X^{\prime}$.

Now let $X=\left\{f_{6}=0\right\}$ be the sextic from proposition 4 . We set

$$
f_{6}^{\prime \prime}:=f_{6}+\varepsilon f_{6}^{\prime}
$$

resp. $X^{\prime \prime}=\left\{f_{6}^{\prime \prime}=0\right\}$. Then

$$
X^{\prime \prime} \cap Q=X \cap Q=L \cup 2 K \cup R .
$$

Moreover for general $\varepsilon$ the sextic $X^{\prime \prime}$ is smooth and the equations

$$
\alpha_{X^{\prime \prime}}(s)\left(P_{i}\right)=0 \quad(i=1, \ldots, 5)
$$

are linearly independent, i.e. $X^{\prime \prime}$ fulfills (S1), (S2) and (S3).

Now let $X$ and $F$ be as above. We want to return to our original problem, i.e. define a deformation $\mathscr{X}=\left(X_{t}\right)_{t \in T}$ such that the Chern classes $c_{1}(F), c_{2}(F)$ extend to $\mathscr{X}$, not however $F$. Since $c_{1}(F)=\mathcal{O}_{X}(-3)$ it is clear that $c_{1}(F)$ extends to $\mathscr{X}$. The hard part is to choose $\mathscr{X}$ s.th. $c_{2}(F)$ extends.

Theorem. Let $X$ and $F$ be as above and assume that $X$ fulfills (S1)-(S3). Then there exists a deformation $\mathscr{X}=\left(X_{t}\right)_{t \in T}$ of $X$ s.th.

(i) The Chern classes $c_{1}(F)=\mathcal{O}_{X}(-3)$ and $c_{2}(F)=L$ extend to $X$. 
(ii) F does not extend to $\mathscr{X}$.

Proof. By proposition 5 we can choose a section $0 \neq s \in W \subset H^{0}\left(\mathcal{O}_{1}(6)\right)$ s.th. $s \notin \operatorname{Im} \alpha_{X}$. Since $s\left(P_{i}\right)=0$ for $i=1, \ldots, 5$ we can define a section $s^{\prime} \in H^{0}\left(\mathcal{O}_{L \cup K \cup R}(6)\right)$ by

Using the exact sequence

$$
\begin{aligned}
& \left.s^{\prime}\right|_{L}=s, \\
& \left.s^{\prime}\right|_{K}=\left.s^{\prime}\right|_{R}=0 .
\end{aligned}
$$

$$
0 \rightarrow \mathcal{O}_{Q}(0,1) \rightarrow \mathcal{O}_{Q}(6) \rightarrow \mathcal{O}_{L \cup K \cup R}(6) \rightarrow 0
$$

and the fact that $h^{1}\left(\mathcal{O}_{Q}(0,1)\right)=0$ (Künneth) we can lift $s^{\prime}$ to $Q$ and hence to $\mathbb{P}$. Let $f_{6}^{\prime}$ be such a lift. We then consider the deformation of $X=\left\{f_{6}=0\right\}$ given by

$$
X_{t}=\left\{f_{6}+t f_{6}^{\prime}=0\right\}
$$

Note that by construction $K \cup R \subset X_{t}$ for all $t$ and hence

$$
X_{t} \cap Q=C_{t} \cup K \cup R
$$

where $C_{t}$ is a divisor of bidegree $(1,1)$ and $C_{0}=K+L$. Let

$$
L_{t}=C_{t}-K
$$

Since $L_{0}=L$ it follows that $c_{2}(F)$ extends to $\mathscr{X}=\left(X_{t}\right)_{t \in T}$.

It remains to show that $F$ does not extend. To see this note that the infinitesimal deformation of $X$ defined by $\mathscr{X}$ is given by $\left.f_{6}^{\prime}\right|_{X} \in H^{0}\left(\mathcal{O}_{X}(6)\right)=H^{0}\left(N_{X / P}\right)$. Look at the maps

$$
\begin{aligned}
& H^{0}\left(N_{X / P}\right) \stackrel{\beta}{\longrightarrow} H^{0}\left(\left.N_{X / P}\right|_{L}\right) \\
& \uparrow \alpha_{X} \\
& \\
& H^{0}\left(N_{L / P}\right) .
\end{aligned}
$$

By construction

$$
\beta\left(\left.f_{6}^{\prime}\right|_{X}\right)=s^{\prime} \notin \operatorname{Im} \alpha_{X}
$$

i.e. the deformation $\left(X_{t}\right)_{t \in T}$ contains no infinitesimal deformation of $L$ in $\mathbb{P}[\mathrm{GH}]$, p. 253. In particular $L$ does not extend to $\mathscr{X}$ and hence $F$ does not extend to $\mathscr{X}$ by proposition 3 .

Remark. It is tempting to try and generalise this construction to either hypersurfaces of higher degree in $\mathbb{P}_{4}$ or - more interesting - to hypersurfaces in higher-dimensional projective space. However there does not seen to exist a straightforward generalisation in either case. 


\section{References}

[GH] Ph. Griffiths, J. Harris, Infinitesimal variations of Hodge structure (II), Comp. Math. 50 (1983), $207-265$.

[Kl] S.L.Kleiman, Geometry on Grassmannians and applications to splitting bundles and smoothing cycles, IHES 36 (1969), 281-297.

[La] $H$. Lange, Jacobian surfaces in $\mathbb{P}_{4}$, J. reine angew. Math. 372 (1986), 71-86.

[OSS] C. Okonek, M. Schneider, H. Spindler, Vector bundles on complex projective space, Prog. in Math. 3, Boston 1980.

[Sch] R.L.E.Schwarzenberger, Vector bundles on algebraic surfaces, Proc. London Math. Soc. 11 (1961), 601-622.

Institut für Mathematik, Universität Hannover, Welfengarten 1, D-3000 Hannover 1 Mathematisch Instituut, Rijksuniversiteit Leiden, Postbus 9512, NL-2300 Leiden

Eingegangen 12. Dezember 1989 